\title{
Evaluation of the Campyslide Agglutination Test for Confirmatory Identification of Selected Campylobacter Species
}

\author{
RICHARD L. HODINKA ${ }^{1 *}$ AND PETER H. GILLIGAN ${ }^{1,2}$ \\ Clinical Microbiology-Immunology Laboratories, North Carolina Memorial Hospital, ${ }^{1}$ and Departments of \\ Microbiology-Immunology and Pathology, School of Medicine, University of North Carolina, ${ }^{2}$ \\ Chapel Hill, North Carolina 27514
}

Received 13 July 1987/Accepted 30 September 1987

\begin{abstract}
The utility of a rapid latex slide agglutination test (Campyslide; BBL Microbiology Systems, Cockeysville, Md.) in detecting selected Campylobacter spp. was evaluated and compared with that of conventional identification methods. Isolated colonies suggestive of Campylobacter spp. were tested directly from primary selective media after incubation at $42^{\circ} \mathrm{C}$ under microaerophilic conditions. Stock cultures of Campylobacter jejuni $(n=27)$ and $C$. coli $(n=3)$ were correctly confirmed to the genus level by latex agglutination when tested in pure cultures or isolated from seeded human feces. A total of $\mathbf{5 0}$ fresh clinical isolates of Campylobacter spp. (45 $C$. jejuni and $5 C$. coli) were examined, with complete agreement observed between the latex test and conventional methods. Of 173 non-Campylobacter isolates tested from primary plates, only 1 rough strain of Pseudomonas aeruginosa produced a false-positive result. Although the manufacturer recommends a 30-min antigen extraction, 1 or 5 min was found to be sufficient. Also, confirmation could be achieved within $24 \mathrm{~h}$ of inoculation of clinical specimens, 2 days earlier than with conventional methods.
\end{abstract}

In recent years, Campylobacter spp., particularly Campylobacter jejuni, have been recognized as important human pathogens which cause both acute bacterial gastroenteritis and extraintestinal infections $(1-5,7,8,10,13)$. The incidence of Campylobacter infections in the United States has been estimated at 1 to 2 million cases per year (3). C. jejuni has been isolated from 3 to $14 \%$ of patients with diarrheal illness in Europe and North America $(2,3,5)$ and has been found in every country in which surveillance has been reported $(2-4,6,8)$. Isolation rates for this organism have approached and often surpassed those of Salmonella spp. or Shigella spp. in patients with enteric illness $(4,6,12,14)$. Individuals of all ages have been infected with $C$. jejuni, although infections are most frequent in young children and young adults $(2,3,5,9)$. Campylobacter infections are especially prevalent among college students, with the fraction of stool cultures yielding Campylobacter spp. on U.S. college campuses ranging from 13 to $53 \%$ (14).

Early resolution of the clinical symptoms of acute gastroenteritis and clearance of the organism from stools are dependent upon promptly beginning appropriate antibiotic therapy (1). To do this, rapid determination of the presence of Campylobacter spp. in feces is required. Currently, the definitive identification of Campylobacter spp. by conventional isolation and biochemical techniques requires a minimum of 48 to $72 \mathrm{~h}$. Presumptive identification of these organisms based on Gram staining and oxidase testing requires only 24 to $48 \mathrm{~h}$ to accomplish, but the accuracy of this identification is dependent on the proficiency of the technologist. In many laboratories in which the isolation of Campylobacter spp. is uncommon, the accuracy of this presumptive identification may not be acceptable. We have evaluated the utility of the Campyslide (BBL Microbiology Systems, Cockeysville, Md.) latex agglutination (LA) test in detecting cell wall antigens of selected Campylobacter spp. This test provides a rapid and simple genus-level confirmation of these human pathogens from cultures.

\footnotetext{
* Corresponding author.
}

\section{MATERIALS AND METHODS}

Bacterial isolates. All bacterial strains studied were clinical isolates recovered at North Carolina Memorial Hospital (NCMH) or the Student Health Service (SHS) of the University of North Carolina, Chapel Hill. The organisms were either fresh clinical isolates or isolates which were recovered from stool specimens from patients and stored frozen in horse blood at $-70^{\circ} \mathrm{C}$ until used. A total of 253 fresh clinical isolates and stock cultures, including 80 Campylobacter spp. and 173 non-Campylobacter isolates, were tested by. LA. All Campylobacter isolates were positively identified to the species level by the methods described by Morris and Patton (11). Non-Campylobacter isolates were identified to the species level by Gram staining, catalase and oxidase reactions, and conventional biochemical and physiological methods.

Culture conditions. Fresh stool specimens from patients and stock culture isolates of Campylobacter spp. were inoculated onto campylobacter agar or campylobacter CVA agar (BBL). Incubation was for 24 or $48 \mathrm{~h}$ at $42^{\circ} \mathrm{C}$ under a microaerophilic atmosphere provided by the CampyPak Envelope (BBL) and jar system or the Campy Pouch (BBL) system. All Campylobacter and non-Campylobacter organisms were isolated from the same media and under the same incubation conditions. For experiments involving seeded stools, six human diarrheal stool specimens, confirmed to be negative for Campylobacter spp., were pooled, and 0.9-ml aliquots were inoculated with $0.1 \mathrm{ml}$ of a turbid suspension (McFarland no. 2 standard) of each stock culture isolate of Campylobacter spp. The seeded stools were inoculated onto campylobacter CVA agar by using sterile Dacron-tipped swabs and were incubated for 24 or $48 \mathrm{~h}$ as described above.

Campyslide LA test. The Campyslide test is an LA assay for the confirmatory genus-level identification of the four major Campylobacter pathogens of human illness (C. jejuni, $C$. coli, $C$. laridis, and $C$. fetus subsp. fetus) from cultures. The test is based upon the detection of cell wall antigens found in these species by use of an anti-Campylobacter antibody-coated latex bead suspension. Initially, the 
Campyslide test was assessed with stock strains of Campylobacter spp. in pure cultures or isolated from seeded human feces. The performance of the test was subsequently evaluated with fresh clinical specimens. Both Campylobacter spp. and non-Campylobacter spp. were tested directly from primary plates of Campylobacter selective media. A limited amount of the organism, either as isolated colonies or as confluent growth from these plates, was required to perform the LA test. The LA test was performed in accordance with the instructions of the manufacture with minor modifications. Briefly, bacterial isolates were suspended in $0.2 \mathrm{ml}$ of extraction reagent to achieve a slightly turbid suspension (McFarland no. 0.5 to 1 standard) and were extracted for 1 , 5 , or $30 \mathrm{~min}$ at room temperature. This step was followed by the addition of $0.2 \mathrm{ml}$ of neutralization reagent. The suspension was vortexed thoroughly, and a change in color from yellow to a mid- to deep purple was indicative of neutralization. If the suspension remained yellow, at least 1 additional drop ( 5 to $20 \mu \mathrm{l}$ ) of neutralization reagent was added to neutralize the mixture. The neutralized antigen extracts ( 25 $\mu l)$ were placed onto two circles of a test slide, and 1 drop (25 $\mu l)$ each of reactive latex and control latex was dispensed for each organism tested. The extract and latex were mixed with plastic stirrers, and the test slide was rotated at $100 \mathrm{rpm}$ on a mechanical rotator for 3 to $4 \mathrm{~min}$. The test slide was then read for distinct visible agglutination. If a sample produced weak agglutination, the test slide was rotated for an additional $6 \mathrm{~min}$ before being read again. A positive antigen control and a negative reagent control were tested with each set of specimens. Agglutination was scored as positive, equivocal, or negative. Equivocal results were considered negative.

\section{RESULTS AND DISCUSSION}

Initial studies with stock cultures of 27 strains of $C$. jejuni and 3 strains of $C$. coli were positive by LA when tested in pure cultures or after isolation from seeded human feces (Table 1). Studies on fresh clinical specimens were then performed. The prevalence of Campylobacter spp. between 28 October 1986 and 30 April 1987 was $17.9 \%$ (37 of 207) for SHS, and that for NCMH was $1.6 \%$ (14 of 881). A total of 34 (92\%) C. jejuni and $3(8 \%) C$. coli strains were isolated from SHS, while $12(86 \%) C$. jejuni and $2(14 \%) C$. coli strains were found at $\mathrm{NCMH}$. Of 51 clinical isolates, 50 were correctly confirmed to the genus level by the LA test (Table 1). One Campylobacter strain isolated from NCMH was not tested.

Of 173 non-Campylobacter isolates tested from primary plates, only 1 rough strain of Pseudomonas aeruginosa

TABLE 1. Campyslide test results for stock culture strains and fresh clinical isolates of Campylobacter spp.

\begin{tabular}{lcc}
\hline \multirow{2}{*}{ Organism(s) } & \multicolumn{2}{c}{ No. of positive organisms ${ }^{a}$} \\
\cline { 2 - 3 } & C. jejuni & C. coli \\
\hline Stock strains (pure cultures) & 27 & 3 \\
$\begin{array}{l}\text { Stock strain in seeded feces } \\
\text { (24-h incubation) }\end{array}$ & 16 & 3 \\
$\begin{array}{l}\text { Stock strain in seeded feces } \\
\quad \text { (48-h incubation) }\end{array}$ & 27 & 3 \\
Fresh clinical isolates & 45 & 5 \\
\hline
\end{tabular}

\footnotetext{
${ }^{a}$ All tested organisms were positive.

${ }^{b}$ Some stock strains showed no visible growth in seeded feces after incubation for $24 \mathrm{~h}$. Growth subsequently occurred after an additional $24 \mathrm{~h}$ of incubation.
}

repeatedly produced a positive agglutination result. However, atypical agglutination reactions with either the reactive or control latex or both were observed for eight other isolates of this organism. Reactions of this type were rapid, were atypical in appearance, and occurred while the antigen extracts were being stirred with the antibody-coated latex particles. These reactions could not be consistently reproduced upon repeat testing of the isolated organisms and were considered equivocal results. Table 2 summarizes the results for the non-Campylobacter isolates tested by LA.

Although the manufacturer recommends a 30 -min antigen extraction for the LA test, we found 1 or $5 \mathrm{~min}$ to be sufficient. Antigens extracted for different times from 44 fresh clinical isolates of Campylobacter spp. (24 isolates for 5- and 30-min extraction comparisons; 20 isolates for 1- and 5-min extraction comparisons) performed equally well when tested by LA (Table 3). A total of 83 non-Campylobacter isolates were extracted for 5 and $30 \mathrm{~min} ; 79$ isolates showed negative results at both extraction times, and 4 isolates of $P$. aeruginosa showed atypical agglutination. Three separate test kits were used for this comparison, and 11 of the 83 non-Campylobacter isolates were $P$. aeruginosa. A comparison of 1- and 5-min extraction times revealed negative results for 49 non-Campylobacter isolates and atypical agglutination for 4 isolates of $P$. aeruginosa. Four separate kits were used for this comparison, and 10 of the 53 organisms examined were $P$. aeruginosa.

Four medical technologists in the bacteriology laboratory at $\mathrm{NCMH}$ were asked to independently evaluate the Campyslide test and comment on its performance. In a blind study, they were given five strains of $C$. jejuni and five non-Campylobacter isolates (Escherichia coli, Enterobacter cloacae, Klebsiella pneumoniae, Citrobacter freundii, and $P$. aeruginosa) to test by LA. Extraction times of 1 and 5 min (two technologists for each) were used. All individuals successfully and correctly completed their evaluations. Weakly positive agglutination reactions were observed by one technologist for the $C$. jejuni isolates after the initial 3- to 4-min rotation of the test slide. In accordance with the instructions of the manufacturer, the test slide was rotated for an additional $6 \mathrm{~min}$ before being read again. Strong positive reactions were observed at this time. The $P$. aeruginosa isolate showed various degrees of atypical agglutina-

TABLE 2. Campyslide test results for 173 non-Campylobacter isolates

\begin{tabular}{|c|c|}
\hline Organism & $\begin{array}{l}\text { No. positive/ } \\
\text { no. tested }\end{array}$ \\
\hline Acinetobacter calcoaceticus subsp. anitratus .. & $0 / 4$ \\
\hline Bacteroides fragilis ................................. & $0 / 1$ \\
\hline Citrobacter freundii ... & $0 / 4$ \\
\hline Enterobacter cloacae ...... & $0 / 21$ \\
\hline Enterobacter aerogenes.... & $0 / 7$ \\
\hline Enterobacter agglomerans.. & $0 / 1$ \\
\hline Escherichia coli ............................................. & $0 / 71$ \\
\hline Klebsiella pneumoniae .............................. & $0 / 10$ \\
\hline Klebsiella oxytoca ................................ & $0 / 5$ \\
\hline 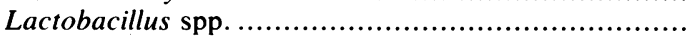 & $0 / 5$ \\
\hline Proteus mirabilis .............. & $0 / 5$ \\
\hline Pseudomonas aeruginosa ................. & $1 / 27^{a}$ \\
\hline Staphylococcus spp. (coagulase negative) .......................... & $0 / 4$ \\
\hline 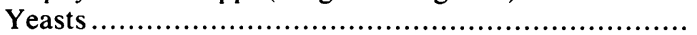 & $0 / 7$ \\
\hline Yersinia enterocolitica....... & $0 / 1$ \\
\hline
\end{tabular}

a One rough strain of $P$. aeruginosa produced a positive agglutination result. Atypical agglutination reactions were observed for eight other isolates of this organism. 
TABLE 3. Detection of Campylobacter isolates by LA following different antigen extraction times

\begin{tabular}{|c|c|c|}
\hline \multirow{2}{*}{ Extraction time $(\mathrm{min})$} & \multicolumn{2}{|c|}{ No. of positive organisms ${ }^{a}$} \\
\hline & C. jejuni & C. coli \\
\hline 30 & $32^{b}$ & $5^{c}$ \\
\hline 5 & 21 & 3 \\
\hline 1 & 19 & 1 \\
\hline
\end{tabular}

${ }^{a}$ All tested organisms were positive.

${ }^{b}$ Eleven isolates of $C$. jejuni were tested by LA following $30 \mathrm{~min}$ of antigen extraction only.

c Two isolates of $C$. coli were tested by LA following $30 \mathrm{~min}$ of antigen extraction only.

tion with the latex test for all of the technologists. The procedure was well received with the exception of the neutralization step. More often than not, the technologists had to add more than an equal volume of neutralization reagent to neutralize the extraction mixture.

All Campylobacter isolates from SHS were confirmed within $24 \mathrm{~h}$ of inoculation of clinical specimens onto selective media, 2 days earlier than with conventional methods routinely used in the bacteriology laboratory at NCMH. Stools from SHS were processed in batches on a given day, immediately placed under strict microaerophilic conditions (CampyPak Envelope or Campy Pouch), and incubated undisturbed for 18 to $24 \mathrm{~h}$ at $42^{\circ} \mathrm{C}$. This procedure was not feasible for the bacteriology laboratory at $\mathrm{NCMH}$, since processing of all specimens is offered on a $24-\mathrm{h}$ basis. Therefore, Campylobacter plates were routinely placed in a candle jar as the stools were processed until they could be transferred to jars with CampyPak Envelopes by day-shift personnel. This procedure may have resulted in a delay of growth of the Campylobacter isolates by an additional $24 \mathrm{~h}$ and, therefore, a subsequent delay in identification. Under these conditions, the Campyslide test would save only 1 day in confirming a positive result for Campylobacter spp. in a clinical specimen.

In conclusion, the Campyslide LA test provides a sensitive and specific test for the confirmatory serological identification of Campylobacter spp. from primary selective media. The test is rapid and simple to perform and, with the exception of an atypical agglutination reaction with some isolates of $\boldsymbol{P}$. aeruginosa, the results are distinct and easily interpreted. The antigen extraction time can be decreased to 1 or 5 min, making the test more suitable for a busy clinical microbiology laboratory. Isolated colonies or confluent growth suggestive of Campylobacter spp. can be tested as early as 18 to $24 \mathrm{~h}$ after incubation of the primary plates, making early detection and prompt administration of antibiotics feasible.

\section{ACKNOWLEDGMENTS}

This work was supported by a grant from BBL Microbiology Systems.

We gratefully acknowledge Kim Wait, Patti Gage, Rebecca Crawford, and Mary Ellen Mangum for their technical expertise. We also thank Karen Alston and Cindy Biles for skillful secretarial assistance.

\section{LITERATURE CITED}

1. Blaser, M. J., I. D. Berkowitz, F. M. LaForce, J. Cravens, L. B. Reller, and W. L. Wang. 1979. Campylobacter enteritis: clinical and epidemiologic features. Ann. Intern. Med. 91:179-185.

2. Blaser, M. J., and L. B. Reller. 1981. Campylobacter enteritis. N. Engl. J. Med. 305:1444-1452.

3. Blaser, M. J., D. N. Taylor, and R. A. Feldman. 1983. Epidemiology of Campylobacter jejuni infections. Epidemiol. Rev. 5: 157-176.

4. Blaser, M. J., J. G. Wells, R. A. Feldman, R. A. Pollard, J. R. Allen, and the Collaborative Diarrheal Disease Study Group. 1983. Campylobacter enteritis in the United States: a multicenter study. Ann. Intern. Med. 98:360-365.

5. Butzler, J. P., and M. B. Skirrow. 1979. Campylobacter enteritis. Clin. Gastroenterol. 8:737-765.

6. Finch, M. J., and L. W. Riley. 1984. Campylobacter infections in the United States: results of an 11-state surveillance. Arch. Intern. Med. 144:1610-1612.

7. Guerrant, R. L., R. G. Lahita, W. C. Winn, and R. B. Roberts. 1978. Campylobacteriosis in man: pathogenic mechanisms and review of 91 bloodstream infections. Am. J. Med. 65:584-592.

8. Karmali, M. A., and P. C. Fleming. 1979. Campylobacter enteritis. Can. Med. Assoc. J. 120:1525-1532.

9. Karmali, M. A., and P. C. Fleming. 1979. Campylobacter enteritis in children. J. Pediatr. 94:527-533.

10. Leuchtefeld, N. W., W. L. Wang, M. J. Blaser, and L. B. Reller. 1981. Campylobacter fetus subsp. jejuni: background and laboratory diagnosis. Lab. Med. 12:481-487.

11. Morris, G. K., and C. M. Patton. 1985. Campylobacter, p. 302-308. In E. H. Lennette, A. Balows, W. J. Hausler, Jr., and H. J. Shadomy (ed.), Manual of clinical microbiology, 4th ed. American Society for Microbiology, Washington, D.C.

12. Riley, L. W., and M. J. Finch. 1985. Results of the first year of national surveillance of campylobacter infections in the United States. J. Infect. Dis. 151:956-959.

13. Skirrow, M. B. 1977. Campylobacter enteritis: a new disease. Br. Med. J. 2:9-11.

14. Tauxe, R. V., M. S. Deming, and P. A. Blake. 1985. Campylobacter jejuni infections on college campuses: a national survey. Am. J. Public Health 75:659-660. 